\title{
A New Algorithm for the Smallest Enclosing Circle
}

\author{
Shang Gao ${ }^{1, ~ a ~}$ and Changbao Wang 2,b \\ ${ }^{1}$ School of Computer Science and Technology, Jiangsu University of Science and Technology, \\ Zhenjiang 212003, China \\ 2 School of Computer Science and Technology, Jiangsu University of Science and Technology, \\ Zhenjiang 212003, China \\ a gao_shang@just.edu.cn, b wangchangbao@126.com \\ * The corresponding author
}

Keywords: Smallest enclosing circle; Computational geometry; Complexity

\begin{abstract}
The minimal enclosing circle is used in planning the location of a shared facility. The feature of the smallest enclosing circle problem is analyzed, and a simple algorithm for the smallest enclosing circle problem is presents. The algorithm is proved and its complexity is discussed. The time complexity

of the algorithm is $O[(2+2 / \Delta) n]$ at the worst case. Numerical experiments show that the algorithm is simple and effective.

\section{Introduction}

The Smallest Enclosing Circle Problem is, simply stated, the problem of finding the smallest circle that completely contains a set of points. The minimal enclosing circle is useful when people want to plan the location of a shared facility. In 1991, Welzl puts forward the new method based on some early research methods. Welzl used randomized incremental algorithm for smallest enclosing ellipse problem, and this algorithm is extended to the smallest enclosing ball problem and the smallest enclosing ellipsoid problem. Mark deBerg improved the method was called the randomized incremental algorithm. In 2005, Frank Nielsen proposed a deterministic algorithm based on dual decision-making, which was called dual decision algorithm. In 2000, Wang Wei et al proposed a fast algorithm for discrete point set minimum bounding circle, known as the most distant point first approximation algorithm. Li Hongjun analysed and compared various algorithms. In this paper, a simple and intuitive method is proposed, the main idea of this paper is find a large circle contains all the points firstly, and then gradually reduced this round, until it cannot be reduced so far.
\end{abstract}

\section{The Smallest Enclosing Circle Problem}

The smallest-circle problem or minimum covering circle problem is a mathematical problem of computing the smallest circle that contains all of a given set of points in the Euclidean plane.

If $n$ is the number of points. The intuitive approach is that all the circles generated by three points and by two points are enumerated, then find the smallest one. The number of circles is $C_{n}^{2}+C_{n}^{3}$, so the complexity of this algorithm is $O\left(n^{3}\right)$.

Most of the geometric approaches for the problem look for points that lie on the boundary of the minimum circle. The smallest enclosing circle has the following properties:

Properties I: Given $N$ points in the plane, the smallest enclosing circle is unique.

If there are two the smallest enclosing circle $C_{1}$ and $C_{2}$, which are the same radius and enclose all points as shown in Fig. 1(a). Then all points are in the intersection part of two circle as shown in Fig. 1. Obviously, you can find the smaller circle $C$, which encloses intersection part as shown in Fig. 1(b). 


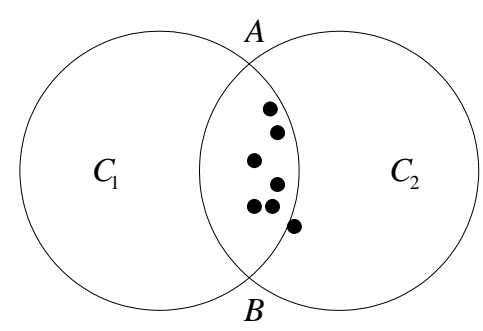

(a) two smallest enclosing circles $C_{1}$ and $C_{2}$

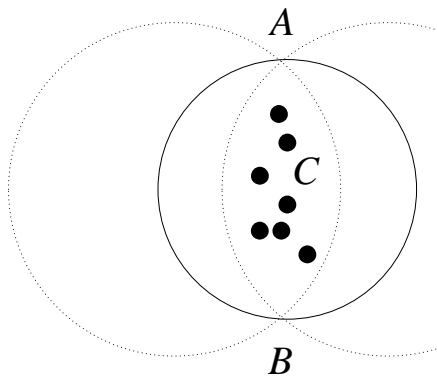

(b) a smaller enclosing circle $C$

Figure 1. Finite The two smallest enclosing circle contained all points

Properties II: The minimum covering circle of a set $\mathrm{S}$ can be determined by at most three points in $\mathrm{S}$ which lie on the boundary of the circle. If it is determined by only two points, then the line segment joining those two points must be a diameter of the minimum circle. If it is determined by three points, then the triangle consisting of those three points is not obtuse.

\section{A new method}

The new method is: At first find a large circle contains all the points, and then gradually reduced this round, until it cannot be reduced so far. Methods are as follows:

Step 1. The center point of these points is as the center of circle. The farthest distance to the center is as the radius as shown in Fig. 2(a). So the circle contains all points.

If only 1 points is on the circle,then go to step 2 . If there are 2 points in the circle, then go to step 3. If there are more than 3 points on a circle, or there are 3 points on a circle and these 3 points of the triangle is acute, the algorithm stop, the circle is the smallest enclosing circle, otherwise go to step 4.
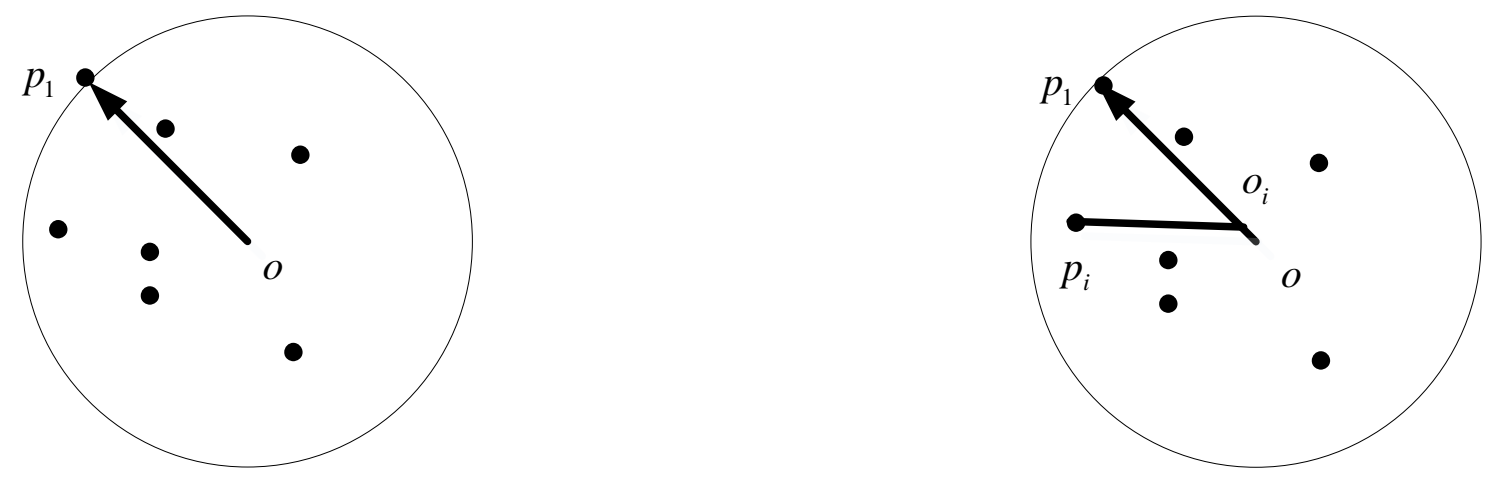

(a) The farthest distance to the center is as the radius (b) The new center $o_{i}$ is $\left(x_{0 i}, y_{0 i}\right)$ and $o_{i}$ is on the $o p_{1}$

Figure 2. Finite One point on the circle

Step 2. The point $\left(x_{1}, y_{1}\right)$ is on the circle. The point $o\left(x_{0}, y_{0}\right)$ is the center. Along the direction $\overrightarrow{o p_{1}}$ reduce radius, until meets second point.

The point $p_{i}$ is $\left(x_{i}, y_{i}\right)$. The new center $o_{i}$ is $\left(x_{0 i}, y_{0 i}\right)$ and $o_{i}$ is on the $o p_{1}$ as the radius as shown in Fig. 2(b). Then

$$
\left\{\begin{array}{l}
x_{0 i}=\alpha x_{1}+(1-\alpha)_{x_{0}} \\
y_{0 i}=\alpha y_{1}+(1-\alpha) y_{0}
\end{array}\right.
$$


Because $o_{i} p_{1}=o_{i} p_{i}$, then

$$
\alpha_{i}=\frac{\left(x_{0}-x_{1}\right)^{2}+\left(y_{0}-y_{1}\right)^{2}-\left(x_{0}-x_{i}\right)^{2}-\left(y_{0}-y_{i}\right)^{2}}{2\left(x_{1}-x_{0}\right)\left(x_{1}-x_{i}\right)+2\left(y_{1}-y_{0}\right)\left(y_{1}-y_{i}\right)}
$$

At this time the radius is $r_{i}=\left(1-\alpha_{i}\right) o p_{1}$. Find $\alpha *=\min _{2 \leq i \leq n-1}\left(\alpha_{i}\right)$. It will be proofed that the circle contains all points as the following.

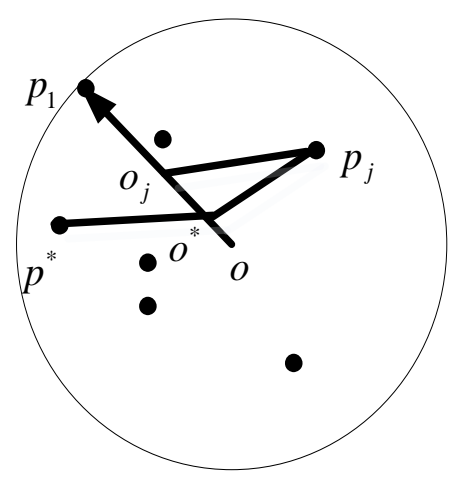

Figure 3. Finite The circle enclose all points which is Determined by the center $o^{*}$

Assume that the radius of $p^{*}$ is the largest. The point $o^{*}$ is center. For any other point, for example $p_{j}$, the center $o_{j}$ must be in the $o^{*} p_{1}$ as shown in Fig. 3. Thus

$$
o^{*} p^{*}=o^{*} p_{1}, o_{j} p_{j}=o_{j} p_{1}
$$

From triangle $\Delta o o_{j} p_{j}$ properties, $O^{*} o_{j}+o_{j} p_{j}>o^{*} p_{j}$. Then

$o^{*} p^{*}=o^{*} p_{1}=o^{*} o_{j}+o_{j} p_{1}=O^{*} o_{j}+o_{j} p_{j}>o^{*} p_{j}$ That is to say That $p_{j}$ is in the circle whose radius is $o^{*} p_{1}$.

If two points $p^{*}$ and $p_{1}$ are on the circle, then the algorithm stops and the circle is the smallest enclosing circle.

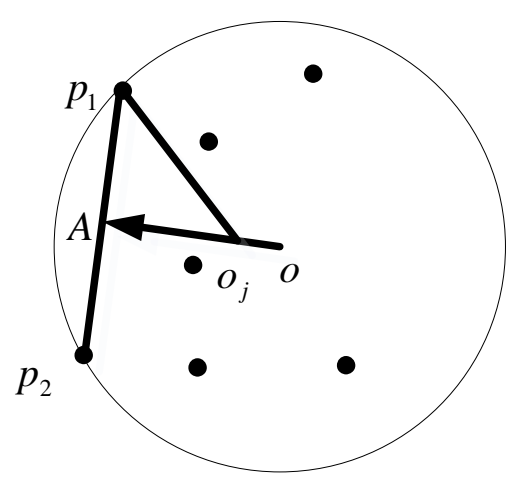

Figure 4. Finite Two points on the circle

Step 3. Assume that there are two points $p_{1}\left(x_{1}, y_{1}\right)$ and $p_{2}\left(x_{2}, y_{2}\right)$ on the circle. The center point $o\left(x_{0}, y_{0}\right)$ is in the perpendicular bisector line of $p_{1} p_{2} . A$ is the midpoint of the line $p_{1} p_{2}$. The center move along the vertical bisector $O A$, and reduce the radius of the circle, until meets the third point as shown in Fig. 4. 
The coordinates of $A$ is

$$
x_{A}=\frac{1}{2}\left(x_{1}+x_{2}\right), \quad y_{A}=\frac{1}{2}\left(y_{1}+y_{2}\right)
$$

Supposed that the new center is $o_{j}\left(x_{0 j}, y_{0 j}\right)$. Because $o_{j}$ is on the $O A$. Thus

$$
\left\{\begin{array}{l}
x_{0 j}=\alpha_{X_{A}}+(1-\alpha)_{X_{0}} \\
y_{0 j}=\alpha y_{A}+(1-\alpha) y_{0}
\end{array}\right.
$$

With $\alpha$ increasing from 0 to $1,{ }^{\mathrm{O}_{j}}$ moves from the center $\mathrm{O}$ to the point $\mathrm{A}$. When $\alpha$ slowly increases, calculated distance of ${ }^{O_{j}}$ to remaining the $n-2$ point. When the distance is greater than or equal to ${ }^{{ }_{j}} p_{1}$, the point is to objection which is found and this point is denoted as $p_{3}$. If the increasing step is $\Delta$, in the worst case, iterative times is $1 / \Delta$. If $\Delta=0.001$, in the worst case, the iterative times is 1000 .

The circumcircle of three points $p_{1}, p_{2}$ and $p_{3}$ is calculated as following.

Supposed that three points coordinates are $\left(x_{1}, y_{1}\right),\left(x_{2}, y_{2}\right)$ and $\left(x_{3}, y_{3}\right)$. Then

$$
\begin{aligned}
& D=\frac{\left(x_{1}^{2}+y_{1}^{2}\right)\left(y_{3}-y_{2}\right)+\left(x_{2}^{2}+y_{2}^{2}\right)\left(y_{1}-y_{3}\right)+\left(x_{3}^{2}+y_{3}^{2}\right)\left(y_{2}-y_{1}\right)}{x_{1}\left(y_{2}-y_{3}\right)+x_{2}\left(y_{3}-y_{1}\right)+x_{3}\left(y_{1}-y_{2}\right)} \\
& E=\frac{\left(x_{1}^{2}+y_{1}^{2}\right)\left(x_{2}-x_{3}\right)+\left(x_{2}^{2}+y_{2}^{2}\right)\left(x_{3}-x_{1}\right)+\left(x_{3}^{2}+y_{3}^{2}\right)\left(x_{1}-x_{2}\right)}{x_{1}\left(y_{2}-y_{3}\right)+x_{2}\left(y_{3}-y_{1}\right)+x_{3}\left(y_{1}-y_{2}\right)} \\
& F=\frac{\left(x_{1}^{2}+y_{1}^{2}\right)\left(x_{3} y_{2}-x_{2} y_{3}\right)+\left(x_{2}^{2}+y_{2}^{2}\right)\left(x_{1} y_{3}-x_{3} y_{1}\right)+\left(x_{3}^{2}+y_{3}^{2}\right)\left(x_{2} y_{1}-x_{1} y_{2}\right)}{x_{1}\left(y_{2}-y_{3}\right)+x_{2}\left(y_{3}-y_{1}\right)+x_{3}\left(y_{1}-y_{2}\right)}
\end{aligned}
$$

The center is $(-D / 2,-E / 2)$.

The radius is $\frac{1}{2} \sqrt{D^{2}+E^{2}-4 F}$.

This circumcircle encloses all the points.

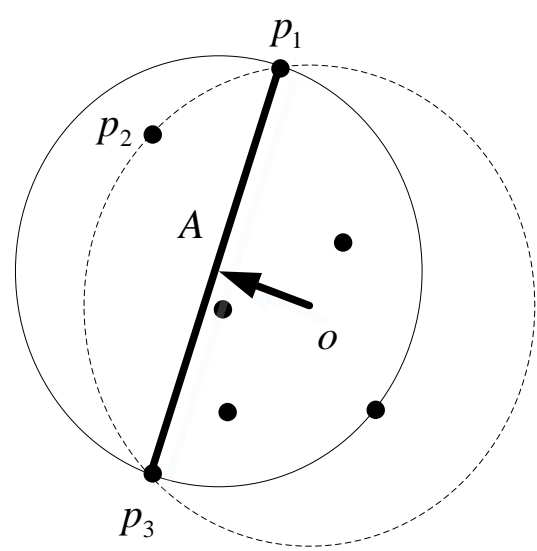

Figure 5. Finite Three points constituting obtuse triangle on the circle

Step 4. Assume that obtuse triangle is constituted by three points $p_{1}, p_{2}$ and $p_{3}$ as shown in Fig. 5. $p_{1} p_{3}$ is largest of obtuse triangle. The center move along the vertical bisector $O A$, and reduce the radius of the circle, until meets the third point as shown in Fig. 4. The calculation process is same as the step 3. 
In the first step of the algorithm, $n$ points are calculated. In the second step, the remaining $n-1$ points are calculated. In the third step, the remaining $n-2$ points are calculated. In the fourth step, the remaining $n-3$ points are calculated. So the worst-case complexity of algorithm is $O[(2+2 / \Delta) n]$.

\section{Example Analysis}

Example 1: The coordinates of 12 points are as shown in table 1 ([6]). The radius is 14.7085 , and the center coordinates is $(18.9786,16.2653)$. the iterative process are shown in Fig. 6

Table 1 coordinates of 12 points

\begin{tabular}{ccc}
\hline point & $\mathrm{x}$ & $\mathrm{y}$ \\
\hline 1 & 15.85 & 6.05 \\
2 & 29.82 & 22.11 \\
3 & 4.84 & 20.32 \\
4 & 25.47 & 29.46 \\
5 & 33.65 & 17.31 \\
6 & 7.30 & 10.43 \\
7 & 28.15 & 6.67 \\
8 & 20.85 & 11.47 \\
9 & 22.83 & 2.07 \\
10 & 26.28 & 23.15 \\
11 & 14.39 & 30.24 \\
12 & 30.24 & 25.23 \\
\hline
\end{tabular}

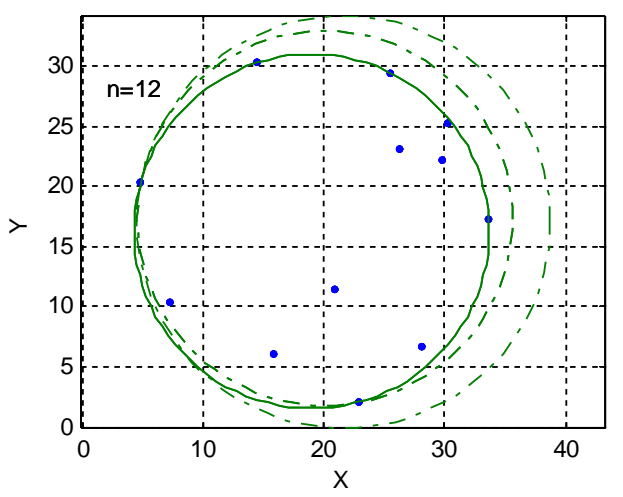

Figure 6. Finite The smallest enclosing circle of 12 points

Example 2: The test data is from ftp://ftp.cs.princeton.edu/pub/Cs226/. The algorithm select the data of Input200.txt and Inpit200.txt. The results are as shown in Fig. 7. 


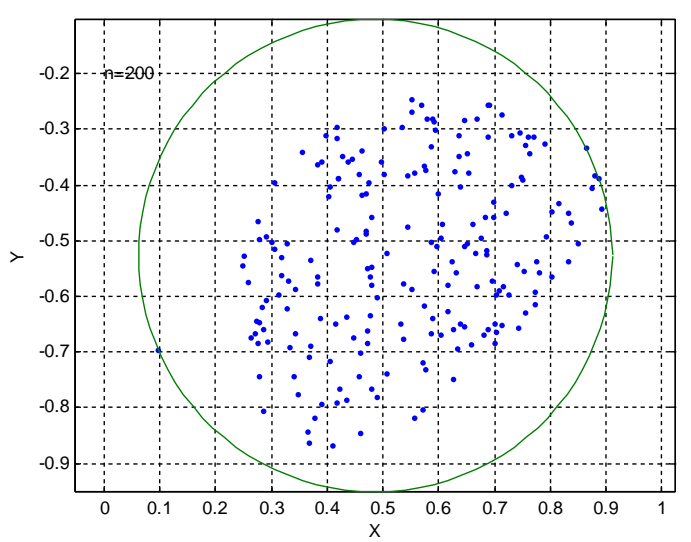

(a) the results of Input200.txt

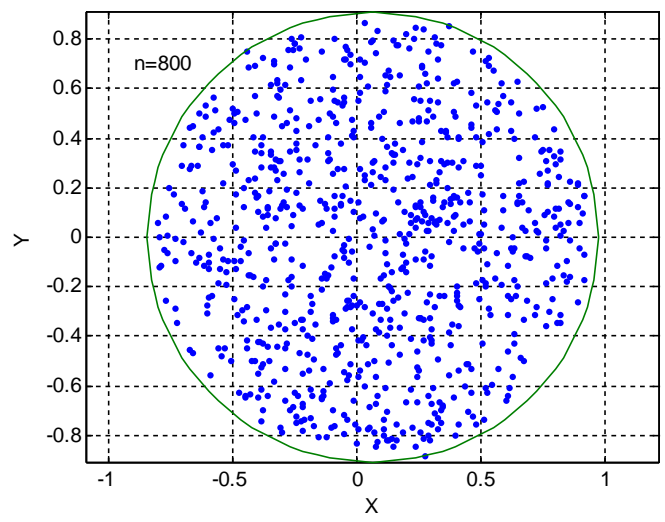

(b) the results of Inpit200.txt

Figure 7. Finite The smallest enclosing circle of Input200.txt and Inpit200.txt

\section{Conclusion}

The smallest enclosing circle problem is an old problem. With the rapid development of computer technology, the smallest enclosing circle problem is applied more and more widely, such as design, application in industrial robot, collision detection, machine learning, computer graphics and other fields. Research results are more and more. This paper presents a simple solution of the smallest enclosing circle algorithm, and give its proof. The algorithm is simple and effective.

\section{References}

[1] W. Emo, "Smallest enclosing disks (balls and ellipsoids)", in Maurer H. (Ed.), New Results and New Trends in Computer Science, Lecture Notes in Computer Science, Heidelberg: Springer-Verlag, vol.555, 1991, pp.359-370.

[2] Mark de Berg, Marc van Kreveld, Mark Overmars, et al, Computational Geometry: Algorithms and Applications. Springer-Verlag, 3rd rev. ed. 2008.

[3] N Frank, N Richard, "A fast deterministic smallest enclosing disk approximation algorithm", Information Processing Letters, 2005, vol.93, no.6, pp.263-268.

[4] W. Wang, W.P Wang, J. Y. Wang, An Algorithm for Finding the Smallest Circle Containing all Points in a Given Point Set", Journal of Software, 2000, vol.11, no.9, pp.1237-1240.(inChinese)

[5] H. J. Li, X. P. Zhang, "Analysis and improvement of smallest enclosing disk algorithm on discrete set of points", JOURNAL OF GRAPHICS, 2012,vol.3, no.2, pp.34-38. (inChinese)

[6] H. F. Wang, J. Liu, "Finding Minimum Contain Circle of Plane Finite Points with Genetic Algorithm", Journal of Nanchang University(Engineering \& Technology), 2007, vol.29, no.4,pp.384-386. (inChinese) 